\title{
Current status of acute myocardial infarction in Korea
}

\author{
Yongcheol Kim ${ }^{1}$, Youngkeun $\mathrm{Ahn}^{1}$, Myeong Chan $\mathrm{Cho}^{2}$, Chong Jin $\mathrm{Kim}^{3}$, Young Jo $\mathrm{Kim}^{4}$, and \\ Myung Ho Jeong ${ }^{1}$
}

\author{
${ }^{1}$ Departemt of Cardiology, \\ Chonnam National University \\ Hospital, Gwangju; ${ }^{2}$ Department \\ of Cardiology, Chungbuk National \\ University Hospital, Cheongju; \\ ${ }^{3}$ Department of Cardiology, Kyung \\ Hee University Hospital, Seoul; \\ ${ }^{4}$ Department of Cardiology, \\ Yeungnam University Medical \\ Center, Daegu, Korea

\section{Received: October 20, 2018} \\ Accepted: November 27, 2018

\section{Correspondence to} \\ Myung Ho Jeong, M.D. \\ Departemt of Cardiology, Chon- \\ nam National University Hos- \\ pital, 42 Jebong-ro, Dong-gu, \\ Gwangju 61469, Korea \\ Tel: $+82-62-220-6243$ \\ Fax: +82-62-228-7174 \\ E-mail: myungho@chollian.net
}

\begin{abstract}
Coronary artery disease, especially acute myocardial infarction (AMI), is a leading cause of death in the Asia-Pacific region. The Korea Acute Myocardial Infarction Registry (KAMIR) is the first nationwide, prospective, multicenter registry of Korean patients with AMI. Since the KAMIR first began in November 2005, more than 70,000 patients have been enrolled, and 230 papers have been published (as of October 2018). Moreover, published data from the KAMIR have revealed different characteristics from those of Western AMI registries regarding risk factors, interventional strategies, and clinical outcomes. As a result, the KAMIR study has improved the outcomes of percutaneous coronary intervention and reduced mortality. We propose the use of the KAMIR score in the prediction of 1-year mortality. Using data from the KAMIR, we provide an overview of the current status of AMI in Korea, including trends in demographic characteristics, risk factors, medications, treatment strategies, and clinical outcomes.
\end{abstract}

Keywords: Myocardial infarction; Risk factors; Percutaneous coronary intervention; Mortality; Prognosis

This paper was contributed by

The Korean Society of Cardiology.

\section{INTRODUCTION}

Although acute myocardial infarction (AMI) continues to be a major cause of mortality in the Asia-Pacific population, there is still a lack of data on the characteristics of patients with AMI in Asia [1]. The Korea Acute Myocardial Infarction Registry (KAMIR) is the first nationwide, prospective, multicenter registry of Korean patients with AMI. The KAMIR provides the public and physicians in the "real-world" clinical field access to the demographic characteristics, treatment strategies, and clinical outcomes of patients with AMI [2]. Since the KAMIR first began in November 2005, more than 70,000 patients have been enrolled, and a total of 230 papers have been published (as of October 2018). Moreover, published data from the KAMIR have revealed different characteristics than those from Western AMI registries $[3,4]$. Using data from the KAMIR, we provide an overview of the current status of AMI in Korea, including trends in demographic characteristics, risk factors, medications, treatment strategies, and clinical outcomes. 


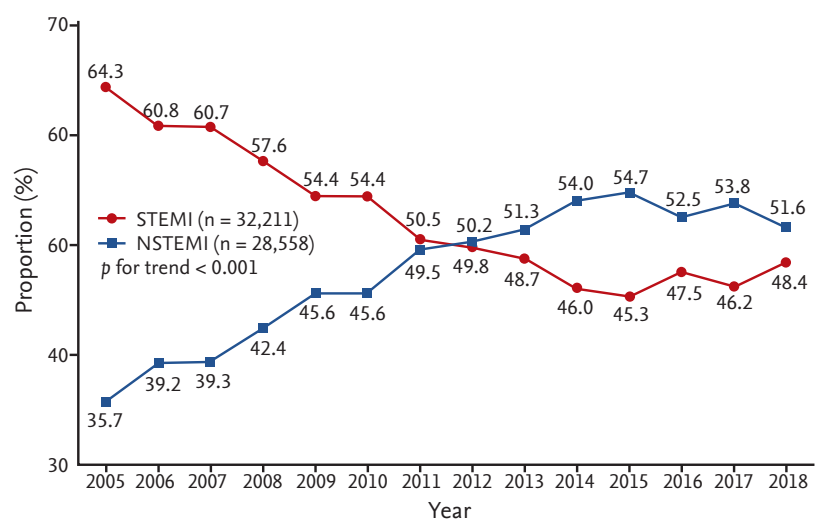

Figure 1. Annual incidence rates of ST-elevation myocardial infarction (STEMI) and non-ST-elevation myocardial infarction (NSTEMI) from 2005 to 2018.
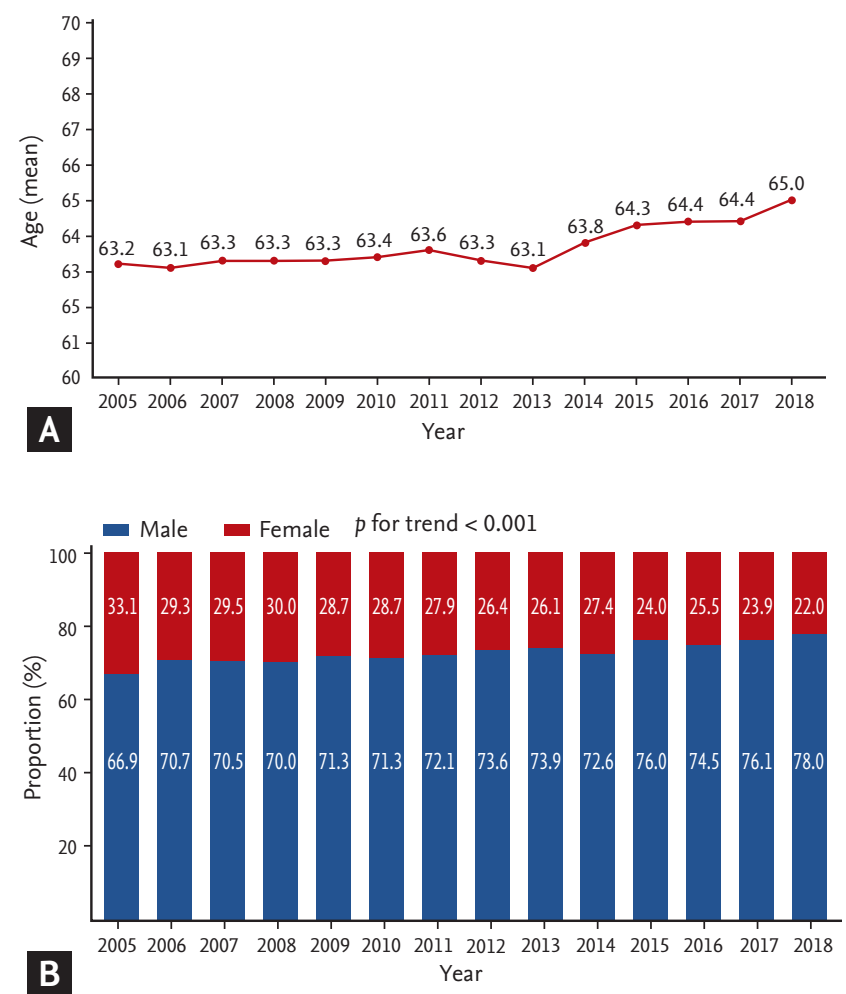

Figure 2. Temporal trends in the (A) mean age and (B) sex ratio among Korean patients with AMI.

\section{DEMOGRAPHIC CHARACTERISTICS IN KOREAN PATIENTS WITH AMI}

Temporal trends in the proportion of patients with AMI, including ST-elevation myocardial infarction (STEMI) and non-ST-elevation myocardial infarction (NSTEMI), are shown in Fig. 1. STEMI decreased from
$64.3 \%$ in 2005 to $48.4 \%$ in 2018 , and thus the ratio of STEMI/NSTEMI decreased in 2012 ( $p$ for trend $<0.001$ ) (Fig. 1). This trend is similar to that reported in previous Western studies $[5,6]$. The change in the STEMI/ NSTEMI ratio can be explained by more sensitive cardiac-specific assays, such as the high-sensitive troponin assay [7-9]. With the wide use of these sensitive tests, the rate of NSTEMI has increased due to the early detection of myocardial necrosis in patients formerly considered to have unstable angina [10]. In only Japan registry, $77 \%$ of all patients with AMI were STEMI and the patients with STEMI increased between 2005 and 2014 [11].

Trends in the mean age and sex ratio of Korean patients with AMI are shown in Fig. 2. Between 2005 and 2018, the mean age of AMI patients gradually increased from 63.2 years in 2005 to 65.0 years in 2018 (Fig. 2A). In terms of the sex ratio, the proportion of males gradually increased from $66.9 \%$ in 2005 to $78.0 \%$ in 2018 ( $p$ for trend $<0.001$ ). In the FAST-MI (French Registry of Acute ST-Elevation or non-ST-elevation Myocardial Infarction), the mean age decreased slightly from 64.0 years in 2005 to 63.5 years in 2015 , and the proportion of males increased from $72.0 \%$ in 2005 to $75.0 \%$ in 2015 [12-14].

\section{PREVALENCE OF CARDIOVASCULAR RISK FACTORS}

Among the cardiovascular risk factors, KAMIR data from November 2005 to August 2018 revealed that hypertension is the most common comorbidity (57.2\%), followed by current smoking (40.6\%), diabetes mellitus (DM; 32.3\%), and dyslipidemia (13.2\%). In the analysis of risk factor trends, the rates of hypertension, DM, and dyslipidemia have gradually increased since 2005 (all p for trend < 0.001) (Fig. 3A). However, the proportion of current smokers among patients with AMI decreased from $43.7 \%$ in 2005 to $36.1 \%$ in 2018 , and this tendency was the same in both STEMI and NSTEMI patients (all $p$ for trend < 0.001) (Fig. 3B). The prevalences of hypertension, DM, and dyslipidemia increased to $75.4 \%$, $41.8 \%$, and $23.0 \%$ in 2018 , respectively. We previously reported that a blood pressure less than 112.2/73.3 $\mathrm{mmHg}$ was associated with worse clinical outcomes because of the U curve phenomenon between blood pressure control and major adverse cardiac events (MACEs) 


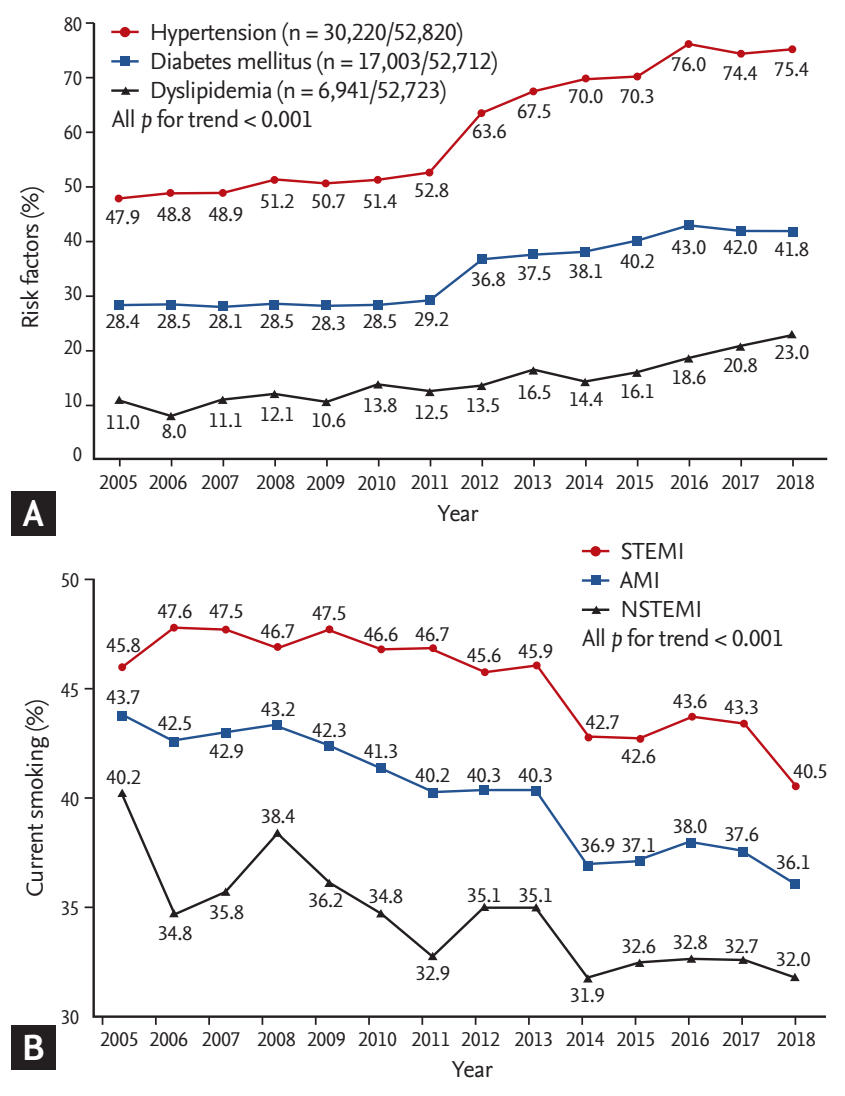

Figure 3. Temporal trends in cardiovascular risk factors among Korean acute myocardial infarction (AMI) patients from 2005 to 2018 . Changes in (A) hypertension, diabetes mellitus, and dyslipidemia in patients with AMI and (B) current smoking in patients with AMI and both ST-elevation myocardial infarction (STEMI) and non-ST-elevation myocardial infarction (NSTEMI).

[15]. In terms of diabetic control, we demonstrated that intensive glycemic control prevented mortality in AMI patients with DM, whereas hypoglycemia should be avoided, as it was associated with increased mortality in AMI patients with DM, especially in the group with poor diabetic control [16]. The rate of dyslipidemia was relatively lower in the KAMIR data than in other Western registries [5,13,14]. This phenomenon could be explained by the different patterns of dyslipidemia in Korean patients with AMI. Approximately $60 \%$ of patients with AMI have low high-density lipoprotein (HDL) levels, and $25 \%$ of those have high triglyceride levels [17]. In addition, although statin therapy for AMI patients with a low-density lipoprotein level less than $70 \mathrm{mg} /$ $\mathrm{dL}$ is proven to be beneficial, combination therapy with simvastatin and ezetimibe was only effective in Korean

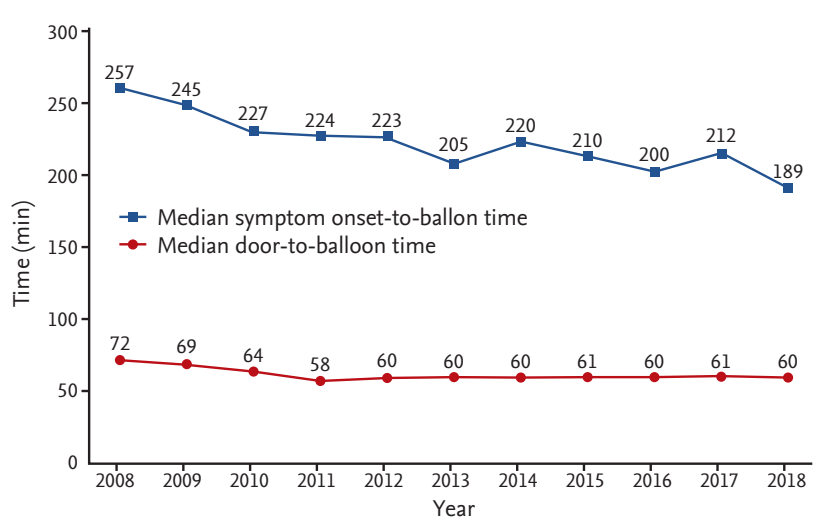

Figure 4. Annual symptom onset-to-balloon time and doorto-balloon time.

AMI patients with high-risk factors, such as old age, $\mathrm{DM}$, and heart failure $[18,19]$. In terms of the current smoking trend in patients with AMI, it is similar to the trend in European registries but remains higher [14,20].

\section{TIME TO PRIMARY PERCUTANEOUS CORONARY INTERVENTION IN STEMI PATIENTS}

The symptom onset-to-balloon ( $\left.\mathrm{S}_{2} \mathrm{~B}\right)$ time and door-toballoon (D2B) time in patients with STEMI are shown in Fig. 4. The S2B time has gradually decreased since 2005, although the D2B time has remained at approximately 60 minutes since 2012. We previously reported the relationship between the time to treatment and 30 day mortality rate among STEMI patients undergoing primary percutaneous coronary intervention (PCI) [21]. Reductions in $\mathrm{S}_{2} \mathrm{~B}$ and $\mathrm{D} 2 \mathrm{~B}$ times did not lead to a parallel reduction in the 30-day mortality rate. In contrast, an $\mathrm{S} 2 \mathrm{~B}$ time of less than 180 minutes was identified as an independent predictor of the 30-day mortality rate. Based on KAMIR data of S2B time, educational programs on the manifestation of STEMI are needed to reduce the duration of out-of-hospital delays in Korea.

\section{PROCEDURAL AND CORONARY ANGIOGRAPHIC CHARACTERISTICS}

According to a previous KAMIR study, radial artery access has markedly increased in Korean patients with 

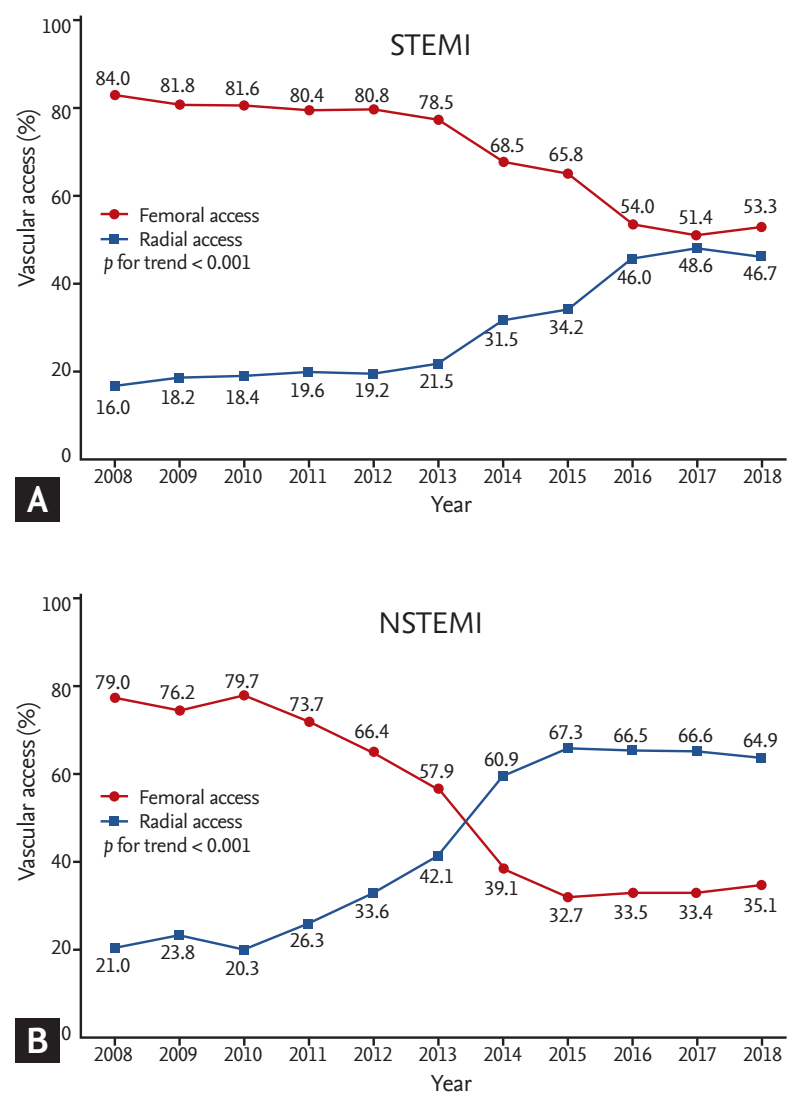

Figure 5. Changing trends in vascular access in patients with (A) ST-elevation myocardial infarction (STEMI) and (B) non-ST-elevation myocardial infarction (NSTEMI) in Korea.

STEMI but has not overtaken femoral access (2016) [4]. This tendency was also observed in extended KAMIR data, even though the rate of radial access has been greater than $50 \%$ in NSTEMI patients since 2014 (Fig. 5). The Minimizing Adverse Haemorrhagic Events by TRansradial Access Site and Systemic Implementation of angioX (MATRIX), a large randomized multicenter trial, demonstrated that radial access is associated with better overall clinical outcomes compared with femoral access in patients with acute coronary syndrome [22,23]. Another KAMIR study also demonstrated that radial access is associated with a lower complication rate and better clinical outcomes in octogenarian patients with AMI [24]. According to the current guidelines, radial access is recommended over femoral access in patients with AMI by an experienced radial operator $[25,26]$. In Korea, although trend analyses have shown an increase in radial access in STEMI patients, the rate did not reach $50 \%$ in 2018 . In NSTEMI patients, radial access

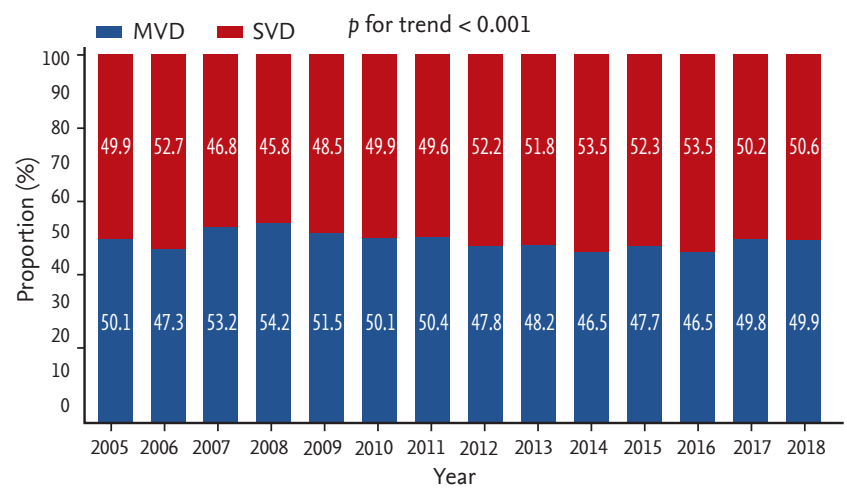

Figure 6. Rates of single-vessel disease (SVD) and multivessel disease (MVD) in patients with ST-elevation myocardial infarction from 2005 to 2018 .

has also remained at approximately $60 \%$ since 2014 . However, radial access is expected to increase in both STEMI and NSTEMI patients due to guideline recommendations.

Multivessel disease (MVD) was observed in half of all STEMI patients in Korea (Fig. 6). Regarding the clinical outcomes of STEMI with MVD, a previous KAMIR study demonstrated that MVD was associated with a significant increase in the 30-day mortality rate [27]. Regarding complete revascularization (CR), there are two types of revascularization [28-34]: one is simultaneous CR, which is defined as simultaneous CR for an infarct-related artery (IRA) and non-IRA during the primary PCI, and the other is staged CR, which is defined as CR with planned PCI for non-IRA within a few weeks after the index procedure, including during index hospitalization. Recent guidelines recommend that any type of CR, including simultaneous or staged CR, should be preferred in STEMI patients with MVD $[25,26,34]$. A 3 -year follow-up single-center retrospective study in Korea demonstrated that staged CR in STEMI patients with MVD improved long-term clinical outcomes without an increase in the rate of repeated PCI [35]. Another study regarding the optimal timing of PCI for non-culprit vessels in patients with STEMI and MVD, the Convergent Registry of Catholic and Chonnam University for Acute Myocardial Infarction (COREA-AMI) registry, showed staged CR after 1 week index primary PCI was associated with the highest MACE, as compared to both simultaneous CR and early staged CR within 1 week [36]. Therefore, simultaneous or staged CR should be considered in Korean patients 


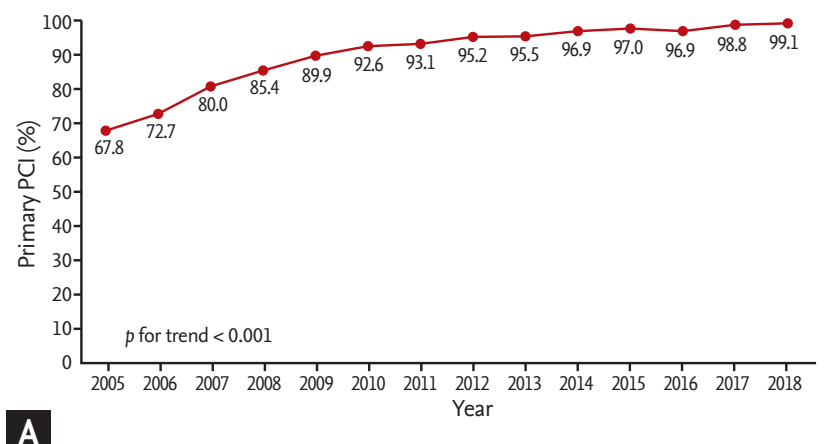

A

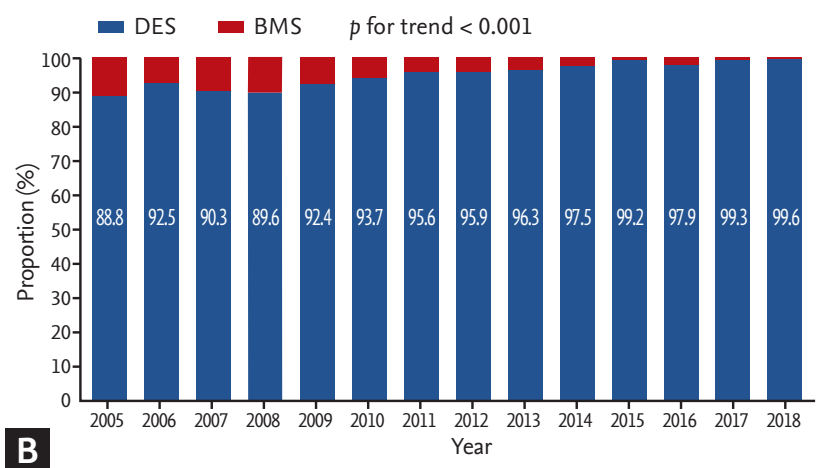

Figure 7. (A) Annual primary percutaneous coronary intervention (PCI) rate and (B) the proportion of drug-eluting stent (DES) and drug-eluting stent (BMS) implantations in patients with ST-elevation myocardial infarction from 2005 to 2018 .

with STEMI and MVD, as per Western guidelines.

Another KAMIR study showed an increased rate of primary PCI and use of drug-eluting stents (DESs) in patients with STEMI between 2005 and 2016 [4]. An extended KAMIR data analysis in 2018 showed a $99.1 \%$ primary PCI rate (Fig. $7 \mathrm{~A}$ ) and 99.6\% DES implantation rate (Fig. 7 B) in STEMI patients. KAMIR data have also shown a notably high rate of primary PCI compared with those in Western registries [14,20].

Several KAMIR studies have reported the safety and efficacy of DESs in patients with AMI [37-40]. A recent KAMIR study demonstrated that the rate of 6-month definite-or-probable stent thrombosis was $0.3 \%$ ( $\mathrm{n}=$ 4) among 1,486 patients with AMI who underwent implantation of a current second-generation DES [41]. Moreover, the rate of definite stent thrombosis at 1 year was $0.6 \%(n=86)$ among 15,003 patients with STEMI who underwent stent implantation between 2005 and 2015 [4]. Therefore, KAMIR studies of DES implantation support the recommendation of implanting a sec- ond-generation DES over a bare-metal stent for primary PCI, as described in the current guidelines $[25,26]$.

\section{MEDICATION AT DISCHARGE}

The medications prescribed to patients with AMI at discharge are shown in Table 1. Statins, renin-angiotensin system (RAS) blockers, and beta blockers are prescribed in approximately $80 \%$ of all AMI patients. RAS blockers, such as angiotensin-converting enzyme inhibitors or angiotensin II receptor blockers (ARBs), are prescribed more for Korean patients with AMI (85.4\%, 86.4\%, and $89.6 \%$ of AMI, STEMI, and NSTEMI patients, respectively) compared with those in French and Swedish registries (64.0\% and 57.0\% of STEMI and NSTEMI patients, respectively, in the French registry and $56.2 \%$ of AMI patients in the Swedish registry) $[20,42]$. A previous KAMIR study demonstrated that insurmountable ARBs, including valsartan, candesartan, irbesartan, telmisartan, and olmesartan, had greater effects on 1-year clinical outcomes than did surmountable ARBs, such as losartan or eprosartan [43]. We also found that ARB therapy at discharge was associated with improved clinical outcomes in STEMI patients with a preserved left ventricular ejection fraction, and that the efficacy of ARBs was comparable with that of angiotensin-converting enzyme inhibitors in STEMI patients with a preserved left ventricular ejection fraction. Therefore, RAS inhibitors should be considered for Korean patients with AMI [44].

In terms of $\mathrm{P}_{2} \mathrm{Y}_{12}$ inhibitors, the use of ticagrelor increased markedly from $10.5 \%$ in 2013 to $46.4 \%$ in 2018 in patients with AMI compared with prasugrel (Fig. 8A). This tendency was also observed in both the STEMI and NSTEMI groups (Fig. $8 \mathrm{~B}$ and $8 \mathrm{C}$ ). In the NSTEMI group, the use of ticagrelor increased from $9.0 \%$ in 2013 to $37.1 \%$ in 2018 , but did not reached above $50.0 \%$. In contrast, in the STEMI group, the use of ticagrelor increased from $12.1 \%$ in 2013 to $55.9 \%$ in 2018 , and ticagrelor use overtook that of clopidogrel in 2016. According to current KAMIR data, the analysis of the trend in $\mathrm{P}_{2} \mathrm{Y}_{12}$ inhibitor use showed that clopidogrel is still prescribed more than $\mathrm{P}_{2} \mathrm{Y}_{12}$ inhibitors despite current guidelines that recommend newer $\mathrm{P}_{2} \mathrm{Y}_{12}$ inhibitors, including ticagrelor and prasugrel, in patients 
Table 1. Medications prescribed to patients with AMI at discharge between 2005 and 2016

\begin{tabular}{|c|c|c|c|c|c|c|c|}
\hline \multirow{2}{*}{ Variable } & \multicolumn{2}{|c|}{$\mathrm{AMI}(\mathrm{n}=54,402)$} & \multicolumn{2}{|c|}{$\operatorname{STEMI}(\mathrm{n}=29,222)$} & \multicolumn{2}{|c|}{$\operatorname{NSTEMI}(\mathrm{n}=25,180)$} & \multirow{2}{*}{$p$ value } \\
\hline & Total no. & No. (\%) & Total no. & No. (\%) & Total no. & No. (\%) & \\
\hline Aspirin & 49,541 & $48,166(97.2)$ & 26,440 & $25,863(97.8)$ & 23,101 & $22,303(96.5)$ & $<0.001$ \\
\hline $\mathrm{P}_{2} \mathrm{Y}_{12}$ inhibitor & 49,249 & $46,039(93.5)$ & 26,353 & $25,270(95.9)$ & 22,896 & $20,769(90.7)$ & $<0.001$ \\
\hline Clopidogrel & 46,039 & $40,986(89.0)$ & 25,270 & $22,487(89.0)$ & 20,769 & $18,499(89.1)$ & 0.780 \\
\hline Ticagrelor & 46,039 & $3,622(7.9)$ & 25,270 & $1,955(7 \cdot 7)$ & 20,769 & $1,667(8.0)$ & 0.250 \\
\hline Prasugrel & 46,039 & $1,431(3.1)$ & 25,270 & $828(3 \cdot 3)$ & 20,769 & $603(2.9)$ & 0.022 \\
\hline Statin & 49,328 & $40,469(82.0)$ & 26,321 & $21,807(82.9)$ & 23,007 & $18,662(81.1)$ & $<0.001$ \\
\hline RAS blocker & 45,685 & $39,012(85.4)$ & 24,479 & $21,161(86.4)$ & 22,985 & $20,603(89.6)$ & $<0.001$ \\
\hline ACE inhibitor & 48,951 & $26,775(54.7)$ & 26,162 & $15,430(59.0)$ & 22,789 & $11,345(49.8)$ & $<0.001$ \\
\hline ARB & 48,628 & $12,647(26.0)$ & 25,911 & $5,949(23.0)$ & 22,717 & $6,698(29 \cdot 5)$ & $<0.001$ \\
\hline Beta-blocker & 49,273 & $38,535(78.2)$ & 26,316 & $20,988(79.8)$ & 22,957 & $17,547(76.4)$ & $<0.001$ \\
\hline Cilostazol & 48,613 & $10,246(21.1)$ & 25,932 & $6,013(23.2)$ & 22,681 & $4,233(18.7)$ & $<0.001$ \\
\hline $\begin{array}{l}\text { Calcium channel } \\
\text { blocker }\end{array}$ & 48,368 & $4,950(10.2)$ & 25,755 & $1,694(6 \cdot 6)$ & 22,613 & $3,256(14 \cdot 4)$ & $<0.001$ \\
\hline Nitrate & 31,805 & $14,036(44.1)$ & 18,018 & $7,740(43 \cdot 0)$ & 13,787 & $6,296(45 \cdot 7)$ & $<0.001$ \\
\hline Nicorandil & 31,658 & $6,805(21.5)$ & 17,956 & $3,981(22.2)$ & 13,702 & $2,824(20.6)$ & 0.001 \\
\hline Spirolactone & 31,285 & $2,469(7.9)$ & 17,749 & $1,427(8.0)$ & 13,536 & $1,042(7 \cdot 7)$ & 0.266 \\
\hline Diuretics & 31,662 & $6,764(21.4)$ & 17,952 & $3,640(20.3)$ & 13,710 & $3,124(22.8)$ & $<0.001$ \\
\hline
\end{tabular}

AMI, acute myocardial infarction; STEMI, ST-elevation myocardial infarction; NSTEMI, non-ST-elevation myocardial infarction; RAS, renin-angiotensin system; ACE, angiotensin-converting enzyme; ARB, angiotensin II receptor blocker. ${ }^{\mathrm{a}}$ STEMI vs. NSTEMI.

with AMI who undergo PCI $[25,26,45]$. A higher rate of clopidogrel use can be explained by previous KAMIR studies regarding the comparison between clopidogrel and the newer $\mathrm{P}_{2} \mathrm{Y}_{12}$ inhibitors. Three KAMIR studies demonstrated that ticagrelor and prasugrel are associated with significantly higher rates of bleeding complications without reducing ischemic events compared with clopidogrel in patients with AMI [46-48]. Regarding the comparison of ticagrelor and prasugrel, another KAMIR study showed that both of these P2Y12 inhibitors showed similar efficacy and safety in patients with STEMI [49]. Therefore, a high bleeding tendency should be considered when prescribing antiplatelet therapy in Korean AMI patients.

\section{TRENDS IN CLINICAL OUTCOMES}

The in-hospital mortality rates are shown in Fig. 9. Between November 2005 and August 2018, the in-hospital mortality rate in patients with AMI decreased gradually from $4.8 \%$ in 2005 to $3.8 \%$ in 2018 . Although this tendency was observed in both the STEMI and NSTEMI groups, it was higher in the former. Furthermore, the rate of in-hospital mortality in STEMI patients was greater than $4.0 \%$ throughout the study period. The 1-year clinical outcomes were analyzed between 2005 and 2015 (Fig. 10). The 1-year rate of MACEs, including all-cause mortality, MI, and any revascularization, decreased from $20.4 \%, 20.5 \%$, and $20.1 \%$ in 2005 to $12.3 \%$, $13.4 \%$, and $11.4 \%$ in 2015 , respectively (Fig. 10A). The 1-year mortality rate also decreased from 2005 to 2015 in both STEMI and NSTEMI patients with AMI (Fig. 10B). The 1-year clinical outcomes improved and were relatively lower than those in Western registries [4]. Mortality is influenced by many factors in AMI patients, including age, comorbidities, and invasive treatment strategies. Higher rates of PCI and primary PCI were observed in KAMIR data relative to Western registries [4]. A high rate of invasive treatment strategies may have led to differences between the KAMIR and Western registries. Moreover, differences in long-term outcomes 

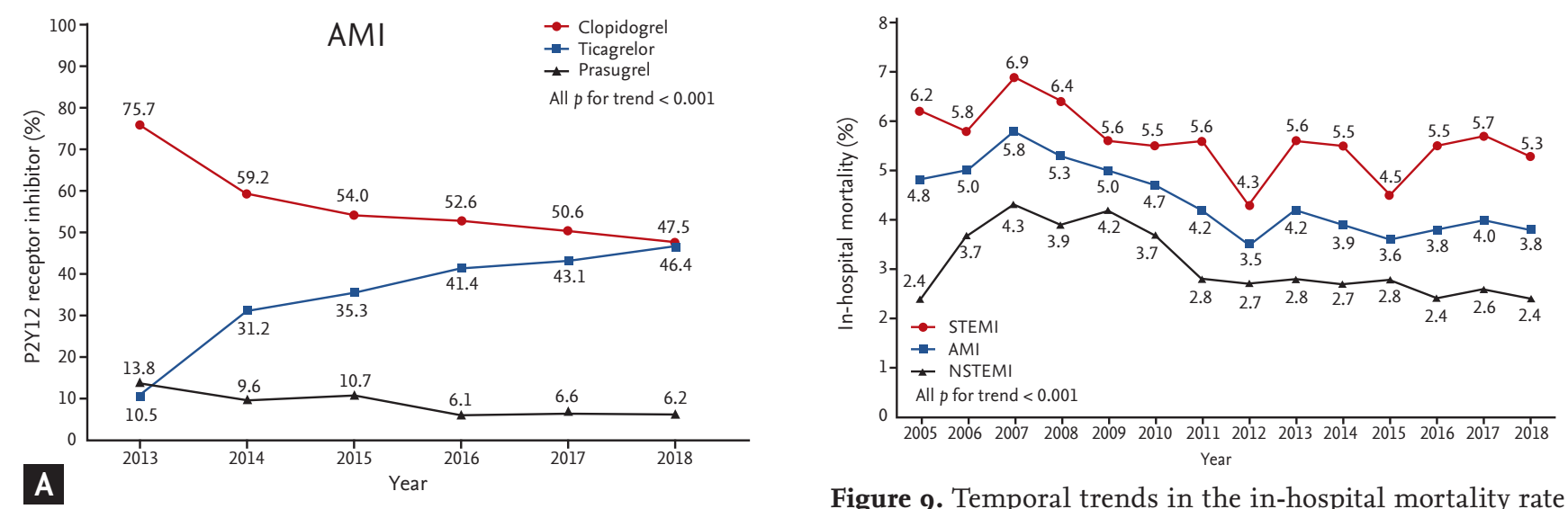

Figure 9. Temporal trends in the in-hospital mortality rate between 2005 and 2018. STEMI, ST-elevation myocardial

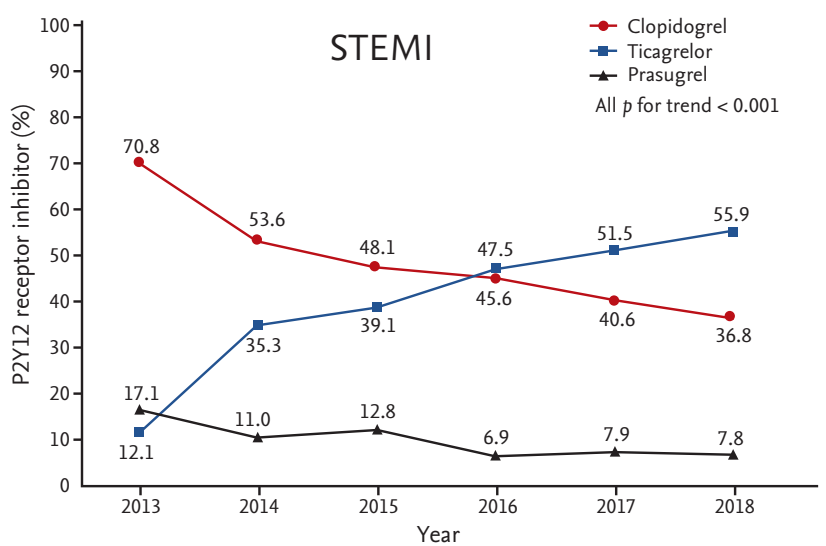
infarction; AMI, acute myocardial infarction; NSTEMI, non-ST-elevation myocardial infarction.

\section{B}

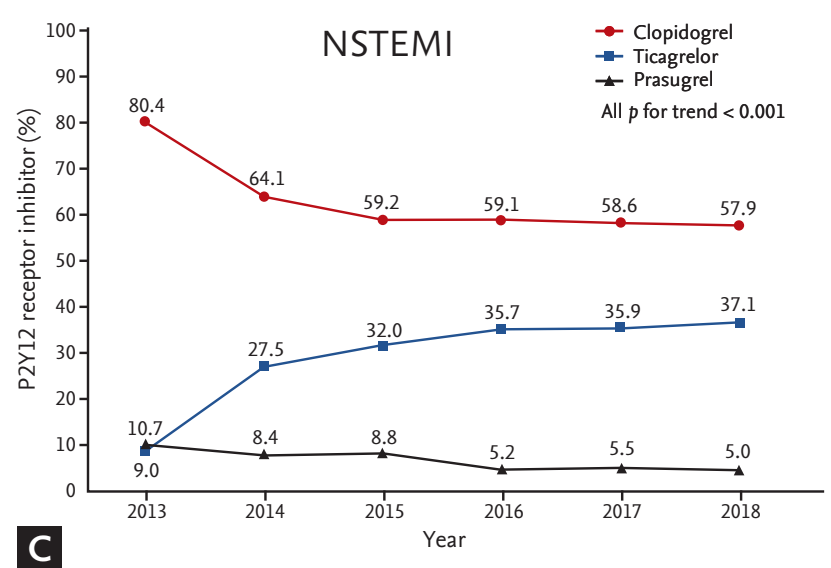

Figure 8. Changing trends in $\mathrm{P}_{2} \mathrm{Y}_{12}$ inhibitors prescribed to patients with (A) acute myocardial infarction (AMI), (B) ST-elevation myocardial infarction (STEMI), and (C) non-ST-elevation myocardial infarction (NSTEMI).

from Western registries led to a new risk score, the KAMIR score, which is more suitable for predicting 1-year mortality in Korean patients with AMI than the Thrombolysis in Myocardial Infarction (TIMI) and Global Reg-

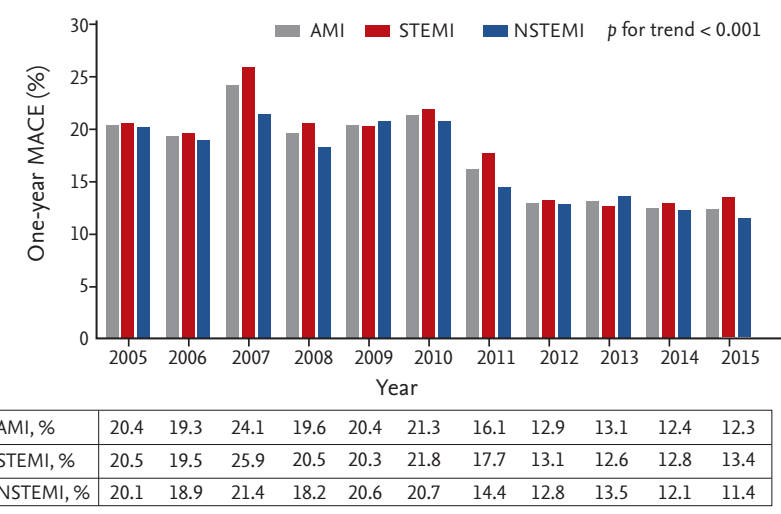

A

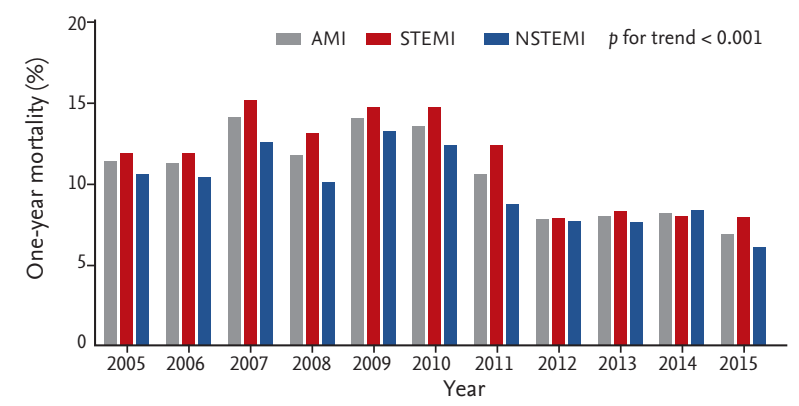

\begin{tabular}{|l|lllllllllll|}
\hline AMI, \% & 11.4 & 11.3 & 14.2 & 11.8 & 14.1 & 13.6 & 10.6 & 7.8 & 8.0 & 8.8 & 6.9 \\
\hline STEMI, \% & 11.9 & 11.9 & 15.2 & 13.2 & 14.7 & 14.8 & 12.4 & 7.9 & 8.3 & 8.8 & 7.9 \\
\hline NSTEMI, \% & 10.6 & 10.4 & 12.6 & 10.1 & 13.3 & 12.4 & 8.7 & 7.7 & 7.6 & 8.4 & 6.1 \\
\hline
\end{tabular}

\section{B}

Figure 10. Temporal trends in (A) 1-year major adverse cardiac event (MACE) and (B) mortality rates from 2005 to 2015. AMI, acute myocardial infarction; STEMI, ST-elevation myocardial infarction; NSTEMI, non-ST-elevation myocardial infarction. 
istry of Acute Coronary Events (GRACE) scores [50,51].

\section{CONCLUSIONS}

Using KAMIR data, we observed several trends in Korean patients with AMI. The rate of STEMI decreased, consistent with Western registries. The mean age and proportion of males also gradually increased in Korean patients with AMI. The prevalences of risk factors, including hypertension, DM, and dyslipidemia, increased, but that of dyslipidemia was lower compared with other Western registries. However, the different patterns of dyslipidemia, low HDL and high triglyceride levels, should be considered in Korean patients with AMI. The D2B time has remained at approximately 60 minutes, but the S2B time has gradually decreased. Although radial artery access has markedly increased in Korean patients with STEMI in recent years, it still remains below 50\%. Regarding STEMI patients with MVD in Korea, simultaneous or staged CR should be considered, as in the current guidelines. In terms of interventional strategies, the rate of PCI has been over 90\% since 2010, and the use of DES implantation in patients with STEMI is notably higher (over 90\% since 2006). Clopidogrel has still high proportion in $\mathrm{P}_{2} \mathrm{Y}_{12}$ inhibitors in patients with AMI who undergo PCI, despite current guidelines recommend newer $\mathrm{P}_{2} \mathrm{Y}_{12}$ inhibitors. In-hospital mortality has decreased among both STEMI and NSTEMI patients with AMI but is still high in STEMI patients (over 4.0\%). The 1-year rates of clinical outcomes, including MACEs and mortality, decreased between 2005 and 2015. KAMIR data can provide physicians with useful information regarding AMI in Korea.

\section{Conflict of interest}

No potential conflict of interest relevant to this article was reported.

\section{Acknowledgments}

This study was performed with the support of the Korean Circulation Society (KCS) as a memorandum of the 5oth Anniversary of the KCS. This study was supported by a grant of Chonnam National University Hospital Biomedical Research Institute (BCRI18015), by a grant of the Korean Health Technology R \& D Project
(HI13 $\mathrm{C}_{1527}$ ), sponsored by the Ministry of Health and Welfare, Republic of Korea.

\section{REFERENCES}

1. OCED, WHO. In-hospital mortality following acute myocardial infarction and stroke. In: Health at a Glance: Asia/ Pacific 2016. Measuring Progress towards Universal Health Coverage. Paris (FR): OECD Publishing, 2016 [cited 2018 Nov 28]. Available from: http://dx.doi.org/10.1787/ health_glance_ap-2016-41-en.

2. Kim Y, Johnson TW, Akasaka T, Jeong MH. The role of optical coherence tomography in the setting of acute myocardial infarction. J Cardiol 2018;72:186-192.

3. Sim DS, Jeong MH. Differences in the Korea Acute Myocardial Infarction Registry compared with Western registries. Korean Circ J 2017;47:811-822.

4. Kim Y, Jeong MH, Ahn Y, et al. Results of a 10-year experience in Korea using drug-eluting stents during percutaneous coronary intervention for acute myocardial infarction (from the Korea Acute Myocardial Infarction Registry). Am J Cardiol 2018;122:365-373.

5. Yeh RW, Sidney S, Chandra M, Sorel M, Selby JV, Go AS. Population trends in the incidence and outcomes of acute myocardial infarction. N Engl J Med 2010;362:2155-2165.

6. Puymirat E, Simon T, Cayla G, et al. Acute myocardial infarction: changes in patient characteristics, management, and 6-month outcomes over a period of 20 years in the FAST-MI Program (French Registry of Acute ST-elevation or non-ST-elevation Myocardial Infarction) 1995 to 2015. Circulation 2017;136:1908-1919.

7. Jaffe AS, Ravkilde J, Roberts R, et al. It's time for a change to a troponin standard. Circulation 2000;102:1216-1220.

8. Antman EM, Grudzien C, Sacks DB. Evaluation of a rapid bedside assay for detection of serum cardiac troponin $\mathrm{T}$. JAMA 1995;273:1279-1282.

9. Apple FS, Wu AH. Myocardial infarction redefined: role of cardiac troponin testing. Clin Chem 2001;47:377-379.

10. Rogers WJ, Frederick PD, Stoehr E, et al. Trends in presenting characteristics and hospital mortality among patients with ST elevation and non-ST elevation myocardial infarction in the National Registry of Myocardial Infarction from 1990 to 2006. Am Heart J 2008;156:1026-1034.

11. Taguchi E, Konami Y, Inoue M, et al. Impact of Killip classification on acute myocardial infarction: data from 
the SAIKUMA registry. Heart Vessels 2017;32:1439-1447.

12. Cambou JP, Simon T, Mulak G, Bataille V, Danchin N. The French registry of Acute ST elevation or non-ST-elevation Myocardial Infarction (FAST-MI): study design and baseline characteristics. Arch Mal Coeur Vaiss 2007;100:524-534.

13. Hanssen M, Cottin $Y$, Khalife K, et al. French Registry on Acute ST-elevation and non ST-elevation Myocardial Infarction 2010: FAST-MI 2010. Heart 2012;98:699-705.

14. Belle L, Cayla G, Cottin Y, et al. French Registry on Acute ST-elevation and non-ST-elevation Myocardial Infarction 2015 (FAST-MI 2015). Design and baseline data. Arch Cardiovasc Dis 2017;110:366-378.

15. Park H, Hong YJ, Cho JY, et al. Blood pressure targets and clinical outcomes in patients with acute myocardial infarction. Korean Circ J 2017;47:446-454.

16. Lee SA, Cho SJ, Jeong MH, et al. Hypoglycemia at admission in patients with acute myocardial infarction predicts a higher 30-day mortality in patients with poorly controlled type 2 diabetes than in well-controlled patients. Diabetes Care 2014;37:2366-2373.

17. Kim KH, Kim CH, Jeong MH, et al. Differential benefit of statin in secondary prevention of acute myocardial infarction according to the level of triglyceride and high density lipoprotein cholesterol. Korean Circ J 2016;46:324-334.

18. Lee $\mathrm{KH}$, Jeong MH, Kim HM, et al. Benefit of early statin therapy in patients with acute myocardial infarction who have extremely low low-density lipoprotein cholesterol. J Am Coll Cardiol 2011;58:1664-1671.

19. Ji MS, Jeong MH, Ahn YK, et al. Clinical outcome of statin plus ezetimibe versus high-intensity statin therapy in patients with acute myocardial infarction propensity-score matching analysis. Int J Cardiol 2016;225:50-59.

2o. Chung SC, Gedeborg R, Nicholas O, et al. Acute myocardial infarction: a comparison of short-term survival in national outcome registries in Sweden and the UK. Lancet 2014;383:1305-1312.

21. Kim HK, Jeong MH, Ahn Y, et al. Relationship between time to treatment and mortality among patients undergoing primary percutaneous coronary intervention according to Korea Acute Myocardial Infarction Registry. J Cardiol 2017;69:377-382.

22. Valgimigli M, Gagnor A, Calabro P, et al. Radial versus femoral access in patients with acute coronary syndromes undergoing invasive management: a randomized multicentre trial. Lancet 2015;385:2465-2476.

23. Valgimigli M, Frigoli E, Leonardi S, et al. Radial versus femoral access and bivalirudin versus unfractionated hep- arin in invasively managed patients with acute coronary syndrome (MATRIX): final 1-year results of a multicentre, randomised controlled trial. Lancet 2018;392:835-848.

24. Lee HW, Cha KS, Ahn J, et al. Comparison of transradial and transfemoral coronary intervention in octogenarians with acute myocardial infarction. Int J Cardiol 2016; 202:419-424.

25. Ibanez B, James S, Agewall S, et al. 2017 ESC guidelines for the management of acute myocardial infarction in patients presenting with ST-segment elevation: the Task Force for the management of acute myocardial infarction in patients presenting with ST-segment elevation of the European Society of Cardiology (ESC). Eur Heart J 2018;39:119-177.

26. Neumann FJ, Sousa-Uva M, Ahlsson A, et al. 2018 ESC/ EACTS guidelines on myocardial revascularization. Eur Heart J 2018.

27. Park DW, Clare RM, Schulte PJ, et al. Extent, location, and clinical significance of non-infarct-related coronary artery disease among patients with ST-elevation myocardial infarction. JAMA 2014;312:2019-2027.

28. Toma M, Buller CE, Westerhout CM, et al. Non-culprit coronary artery percutaneous coronary intervention during acute ST-segment elevation myocardial infarction: insights from the APEX-AMI trial. Eur Heart J 2010; 31:1701-1707.

29. Hannan EL, Samadashvili Z, Walford G, et al. Culprit vessel percutaneous coronary intervention versus multivessel and staged percutaneous coronary intervention for ST-segment elevation myocardial infarction patients with multivessel disease. JACC Cardiovasc Interv 2010;3:22-31.

30. Task Force on the management of ST-segment elevation acute myocardial infarction of the European Society of Cardiology (ESC), Steg PG, James SK, et al. ESC guidelines for the management of acute myocardial infarction in patients presenting with ST-segment elevation. Eur Heart J 2012;33:2569-2619.

31. American College of Emergency Physicians; Society for Cardiovascular Angiography and Interventions, O'Gara PT, et al. 2013 ACCF/AHA guideline for the management of ST-elevation myocardial infarction: a report of the American College of Cardiology Foundation/American Heart Association Task Force on Practice Guidelines. J Am Coll Cardiol 2013;61:e78-e140.

32. Wald DS, Morris JK, Wald NJ, et al. Randomized trial of preventive angioplasty in myocardial infarction. N Engl J Med 2013;369:1115-1123.

33. Gershlick AH, Khan JN, Kelly DJ, et al. Randomized trial 
of complete versus lesion-only revascularization in patients undergoing primary percutaneous coronary intervention for STEMI and multivessel disease: the CvLPRIT trial. J Am Coll Cardiol 2015;65:963-972.

34. Levine GN, Bates ER, Blankenship JC, et al. 2015 ACC/ AHA/SCAI focused update on primary percutaneous coronary intervention for patients with ST-elevation myocardial infarction: an update of the 2011 ACCF/AHA/SCAI guideline for percutaneous coronary intervention and the 2013 ACCF/AHA guideline for the management of ST-elevation myocardial infarction. J Am Coll Cardiol 2016;67:1235-1250.

35. Kim MC, Jeong MH, Park KH, et al. Three-year clinical outcomes of staged, ad hoc and culprit-only percutaneous coronary intervention in patients with ST-segment elevation myocardial infarction and multivessel disease. Int J Cardiol 2014;176:505-507.

36. Kim I, Kim MC, Jeong HC, et al. Optimal timing of percutaneous coronary intervention for nonculprit vessel in patients with ST-segment elevation myocardial infarction and multivessel disease. Korean Circ J 2017;47:36-43.

37. Hong YJ, Jeong MH, Ahn Y, Kang JC. The efficacy and safety of drug-eluting stents in patients with acute myocardial infarction: results from Korea Acute Myocardial Infarction (KAMIR). Int J Cardiol 2013;163:1-4.

38. Ahmed $\mathrm{K}$, Jeong $\mathrm{MH}$, Chakraborty $\mathrm{R}$, et al. Comparison of zotarolimus- and everolimus-eluting stents in patients with ST-elevation myocardial infarction and chronic kidney disease undergoing primary percutaneous coronary intervention. J Cardiol 2014;64:273-278.

39. Piao ZH, Jeong MH, Li Y, et al. Comparison of second-generation drug-eluting versus bare-metal stents in octogenarian patients with ST-segment elevation myocardial infarction. Int J Cardiol 2014;177:1081-1084.

40. Ji MS, Jeong MH, Ahn YK, et al. Comparison of Resolute zotarolimus-eluting stents versus everolimus-eluting stents in patients with metabolic syndrome and acute myocardial infarction: propensity score-matched analysis. Int J Cardiol 2015;199:53-62.

41. Kim Y, Oh SS, Jeong MH, et al. Comparison of short-term clinical outcomes between Resolute Onyx zotarolimus-eluting stents and everolimus-eluting stent in patients with acute myocardial infarction: results from the Korea Acute Myocardial infarction Registry (KAMIR). Cardiol J 2018.

42. Szummer K, Wallentin L, Lindhagen L, et al. Improved outcomes in patients with ST-elevation myocardial infarction during the last 2oyears are related to implementation of evidence-based treatments: experiences from the SWEDEHEART registry 1995-2014. Eur Heart J 2017;38:3056-3065.

43. Jeong HC, Jeong MH, Ahn Y, et al. Comparative assessment of angiotensin II type 1 receptor blockers in the treatment of acute myocardial infarction: surmountable vs. insurmountable antagonist. Int J Cardiol 2014;170:291-297.

44. Yang JH, Hahn JY, Song YB, et al. Angiotensin receptor blocker in patients with ST segment elevation myocardial infarction with preserved left ventricular systolic function: prospective cohort study. BMJ 2014;349:g6650.

45. Valgimigli M, Bueno H, Byrne RA, et al. 2017 ESC focused update on dual antiplatelet therapy in coronary artery disease developed in collaboration with EACTS: the task force for dual antiplatelet therapy in coronary artery disease of the European Society of Cardiology (ESC) and of the European Association for Cardio-Thoracic Surgery (EACTS). Eur Heart J 2018;39:213-26o.

46. Park KH, Jeong MH, Ahn Y, et al. Comparison of shortterm clinical outcomes between ticagrelor versus clopidogrel in patients with acute myocardial infarction undergoing successful revascularization; from Korea Acute Myocardial Infarction Registry-National Institute of Health. Int J Cardiol 2016;215:193-200.

47. Park KH, Jeong MH, Kim HK, et al. Comparison of prasugrel versus clopidogrel in Korean patients with acute myocardial infarction undergoing successful revascularization. J Cardiol 2018;71:36-43.

48. Kang J, Han JK, Ahn Y, et al. Third-generation P2Y12 inhibitors in East Asian acute myocardial infarction patients: a nationwide prospective multicentre study. Thromb Haemost 2018;118:591-600.

49. Kim MC, Jeong MH, Sim DS, et al. Comparison of clinical outcomes between ticagrelor and prasugrel in patients with ST-segment elevation myocardial infarction: results from the Korea Acute Myocardial Infarction Registry-National Institutes of Health. Circ J 2018;82:1866-1873.

50. Kim HK, Jeong MH, Ahn Y, et al. A new risk score system for the assessment of clinical outcomes in patients with non-ST-segment elevation myocardial infarction. Int J Cardiol 2010;145:450-454.

51. Kim HK, Jeong MH, Ahn Y, et al. Hospital discharge risk score system for the assessment of clinical outcomes in patients with acute myocardial infarction (Korea Acute Myocardial Infarction Registry [KAMIR] score). Am J Cardiol 2011;107:965-971. 